\title{
Design of Sub-regional Lighting Control System for Classroom
}

\author{
Jiang-bo Chen, Rui Xue, Pei-xue Liuªnd Xiao Zhang \\ Qingdao Huanghai University, Qingdao, Shandong, China \\ ajiangbochen1981@163.com
}

\begin{abstract}
The sub-regional multi mode lighting control system for classroom is designed to reduce the waste of energy. In the design, RS-485 communication network is used to establish all the area light. Using illumination sensor, vibration sensor and infrared sensor, the solution can automatically collect information of classroom illumination and working state, distribution of people and so on. So that the system can propose a program of switching lights by the chip for data analysis and comparison of data from the overall. Through a certain control algorithm, the control system can achieve good energy saving effect.
\end{abstract}

Keywords: Lighting system, Single-chip, Multi mode, Detector

\section{Introduction}

With the development of economy and the progress of the society, energy demand is increasing and the supply of energy is relatively decreasing. what's more, the prominent energy's wasting makes our countries' energy situation quite grim. And the electric energy which is closely related to people's production and life is very inadequate, so how to improve the utilization rate of electric resource has become a problem which needs to be urgent for solving. In the search for the alternatives to improve energy efficiency and energy saving ways, we found that energy saving is consistent with the requirements of sustainable development. According to the statistics in 2010, the whole society of our country's total electricity consumption of about 24000 million kwh, lighting electricity for about 300 billion kwh, and the annual increase rate of $13 \% \sim 14 \%$, Now the lighting power consumption is more than 500 billion $\mathrm{kwh}$, additional lighting electricity 200 billion $\mathrm{kwh}$. In order to ease the situation of electric tension, improve the classroom lighting efficiency and maximum reduce energy waste has become an inevitable choice, so it has important meaning to research a classroom intelligent lighting control system.

Based on the above situation, this light control system is developed, which machine of STC89C54 is a major part, using integrated optical frequency converter TSL230 to carry out accurate illuminance detection and the environment of development is better than before. This system can satisfy the following functions, such as controlling the circuit of illumination, testing and processing daylight signal, testing and processing the signal of human body that illuminates the back track exists, etc. In order to satisfy these functions, the system adopted the homologous methods respectively, and to the gearing of the system, analyzing how to install, test and run the system.

In the control method, joined the light intensity of upper and lower threshold, which can avoid the mistake operation, was caused by the insufficiency of light intensity lights, system cannot recognize light enough because of the situation is provided by ambient light or by light. Adding the number of thresholds, it canovercome the promble because of the fragmentation of the distribution of the staff to open more light. In data measurement, the system can calculate the illuminance through the output frequency by the directe light frequency converter, no longer with simple strength to define the size of the light intensity, and also realize the control method of adding light intensity threshold upper basis when it have a light switch in a clear standard.

\section{Structure design of the system}

The system adopts the master-slave structure, which consists of two parts: the upper computer and the lower computer. 


\section{Upper computer}

The upper computer mainly to achieve the following functions:

(1) through the keyboard to complete the set password, initialization time, set the threshold of the number of illumination and set the working hours and other tasks.

(2) the control mode is judged by the signal detector.

(3) according to the received lower computer data, and the internal storage of the illumination threshold and the number of threshold calculation control lamp group, and to the corresponding lower machine issued a switch light instructions.

(4) display the current working state and working mode by LCD.

The upper computer design is shown in Fig.1.

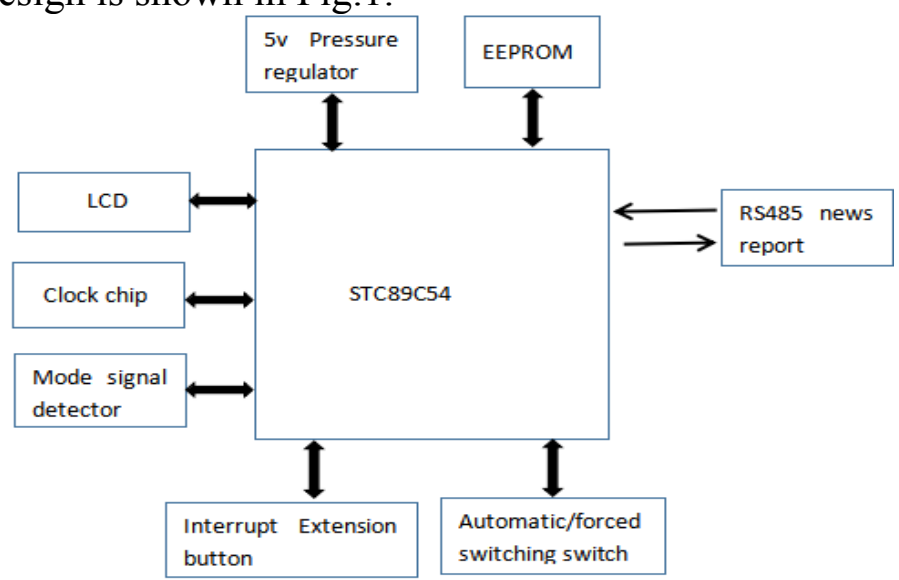

Fig.1 the upper computer connection design

\section{Lower computer}

The Computer curriculum, lighting information stored in the FLASH microcontroller, to achieve power down protection. The system also has the password control, thereby increasing the safety of system operation. Through the keyboard to set the password, initialization time. Clock module uses DS1302 chip, the chip can not only display the second, points, date, and month year of information, but also can realize the power down protection, provides power for the clock, as shown in figure.

The lower computer design is shown in Fig.2.

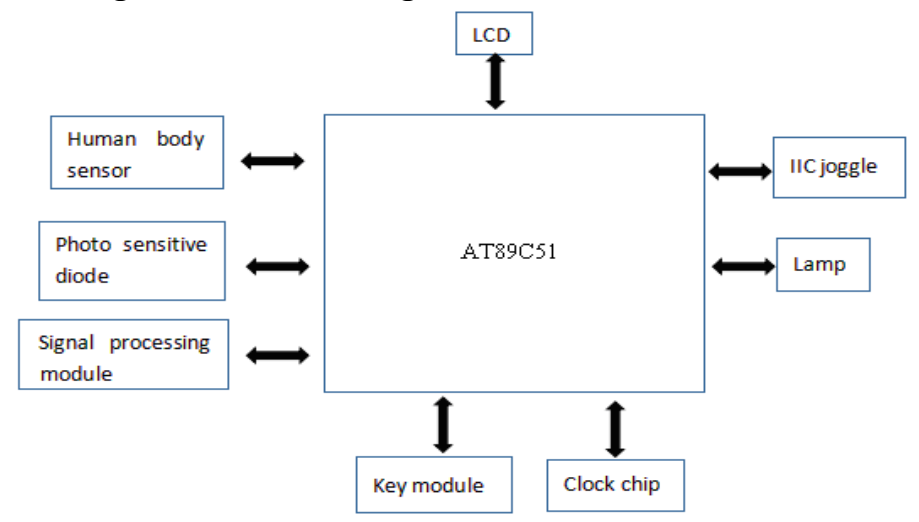

Fig.2 the lower computer connection desig

\section{Hardware design of the system}

\section{Master slave center processing chip}

The main control module of the system uses STC89C54, AT89C51 microcontroller as the core component. These two chips have the advantages of low price, multiple flash, a wide range of applications. STC89C54, AT89C51 microcontroller are also the core of the whole hardware, all other 
circuit module should be connected with them, they are used to process the data in the system, which can connect each module and realize the coordination between the modules. The relation of single chip microcomputer and other circuit modules is described above.

\section{Display module and key module}

In order to facilitate the initialization of the system, this design select the parameter settings and the host mode, this mode is a good way to configure LCD display, key module is made up of four key. The display part uses LCD with Chinese font display module LM3033. Four keys are connected to the microcontroller by an external interrupt expansion. As a result of the limitation of liquid crystal display, the design uses a split screen display system. When setting the information on the screen, need to use buttons to control the cursor, In the parameter Settings, use the button to increase or reduce the control values.

Through the visual face illumination threshold system, personnel and work time threshold settings, the user can according to the actual situation and the need to set the parameters of flexible, can increase the system's availability at the same time, can expand the application range of the system. At the same time, adding a password control interface, form a security enhancement system, the system can clear the user to set permissions.

\section{Optical frequency conversion module}

System adopts programmable optical frequency converter TSL230 as the optical frequency conversion module of the main chip.TSL230 is a new generation of integrated intelligent sensor, photodiode array and current / frequency converter configuration it will be integrated in a monolithic integrated circuit, without external components to complete the light / high resolution frequency conversion the digital signal output, high precision, is a high performance, low-cost intelligent sensor. The output duty ratio of $50 \%$ square wave, and the output frequency and intensity is linear, the sensitivity is adjustable. The functional block diagram is shown as Fig.3.

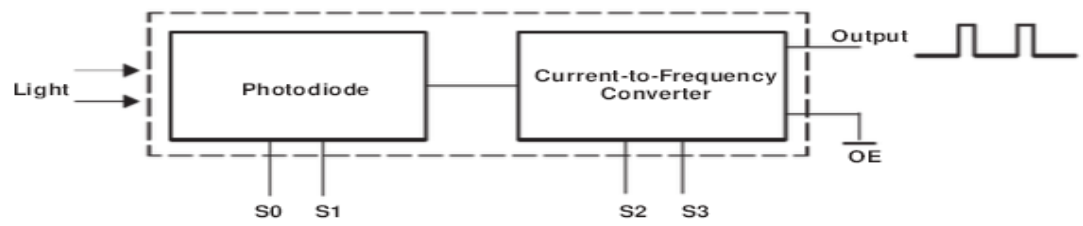

Fig.3 Functional block diagram

The interface circuit of the TSL230and the AT89C51 is shown in Figure 5. TSL230 chip converts light intensity to the corresponding voltage signal pulse, but the voltage signal amplitude changes too small, then the sensor output signal is fed into the 74HC14 chip, which can change the input slow change signal into a clear, jitter free output signal. then $74 \mathrm{HC} 14$ chip plastic the input signal, improve the steepness of the edge of the sign, reduce the frequency count error of the signal. The connection diagram of TSL230 with 74HC14 is shown as Fig.4.

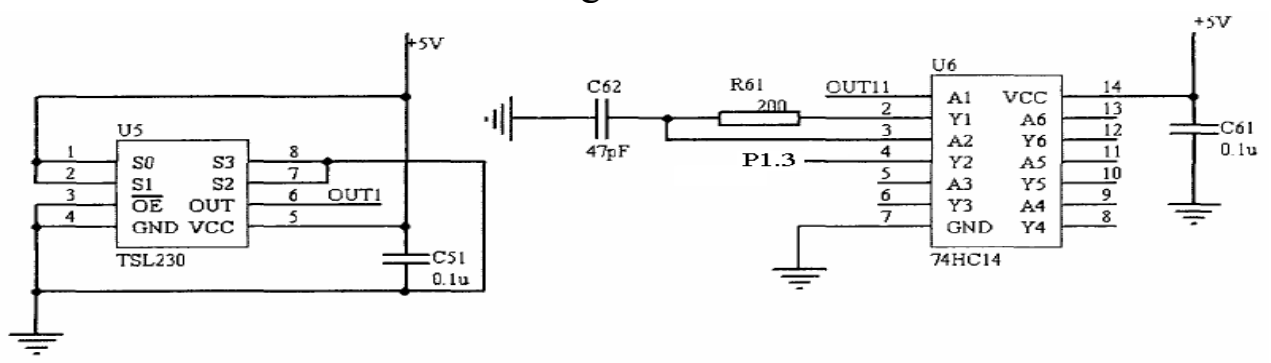

Fig.4 Connection diagram of TSL230 with $74 \mathrm{HC} 14$

\section{Infrared pyroelectric human body detection module}

A pyroelectric infrared sensor is used in the infrared detection module of the system, the sensor is mainly made of a high pyroelectric coefficient of broad-spectrum materials, The size of detector element is $2 \mathrm{~mm} \times 1 \mathrm{~mm}$, wavelength sensitivity of the sensor is in the range between $0.2 \mu$ to $20 \mu$, 
while the body temperature is around 37 degrees commonly fluctuate within a small scale, the human body central wavelength of infrared radiation ranges from $9 \mu$ to $10 \mu$, In order to make the pyroelectric infrared sensor detection sensitivity is as far as possible in the scope of the system needs, we need install the interference filter in the sensor window, thus the infrared sensor is just right for detecting human body radiation.

Because the output signal waveform of pyroelectric infrared sensor changes slowly, the output signal amplitude of pyroelectric infrared sensor is small, The signal processor BIS0001 is used to complete the processing of the pyroelectric sensor output signal. The BIS0001 chip has two working modes, when the pin A is set to 1, the BIS0001 chip is repeatable trigger mode, on the contrary, the BIS0001 chip is not repeatable trigger mode. The chip in this system adopts repeatable trigger mode, The signal processing circuit of pyroelectric infrared sensor is shown as Fig5.

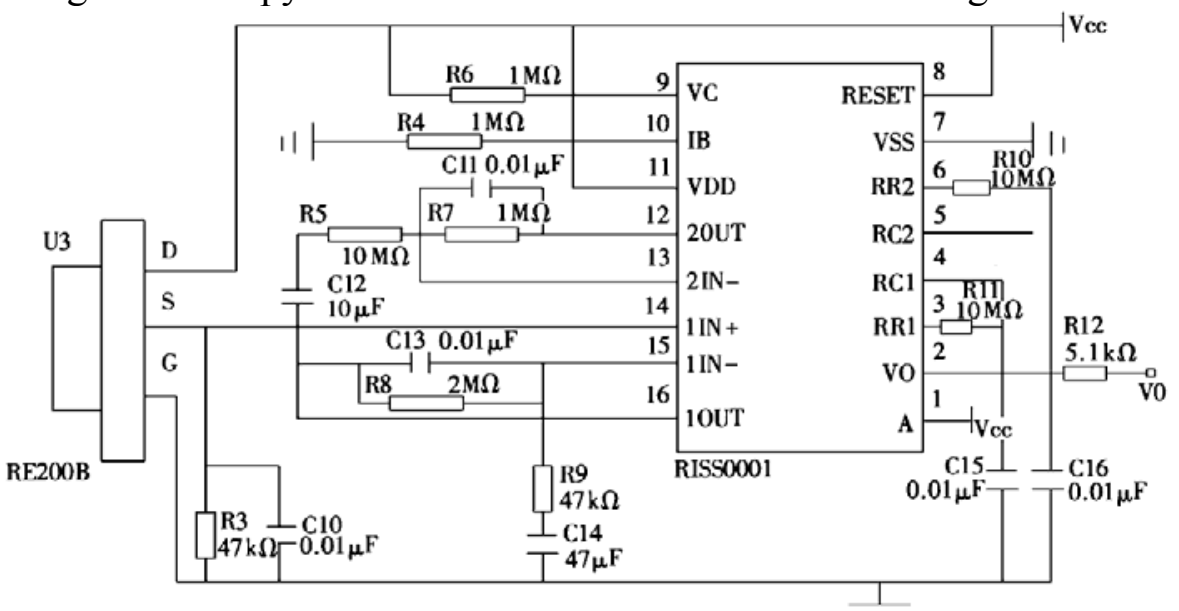

Fig.5 Signal processing circuit of pyroelectric infrared sensor

The connection between the infrared human body induction module and microcontroller is shown as Fig6,Power supply voltage to the infrared sensor after LM7808 and capacitance voltage filtering. The output signal is connected with the resistance of $10 \mathrm{k} \Omega$, and finally the output signal is connected with the I/O interface P1.0 of the AT89C51, and finally realizes the detection of the output state of the sensor.

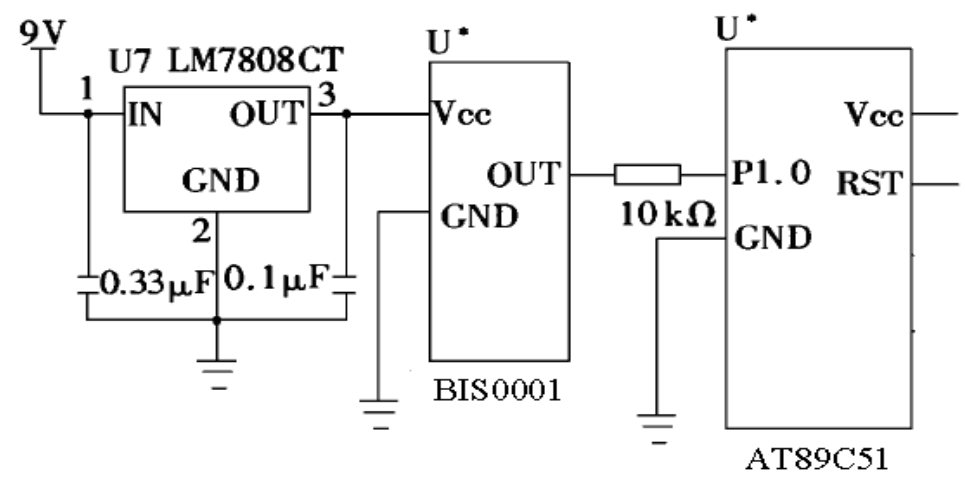

Fig. 6 the connection diagram of Infrared human body induction module and microcompute

\section{Software design of the system}

The system adopts the multiple mode control, in order to meet the needs of the various functions of the classroom mode, mainly divided into: compulsory and automatic modes; automatic mode is subdivided into: multimedia mode, writing mode and study mode. During working hours, if the mode signal input system into the corresponding mode; if not then entered the study no mode. During the work time, such as a compulsory manual input signal, is set according to the operation manual tasks; if not then turn off all the lights.

When the machine is in the self-study mode, the input parameters for the existence of the human body and the light intensity signal, if the human body sensor in a region of the detected effective signal system to judge at optical frequency converter the collected light intensity, if higher than the 
set threshold, then open the corresponding area of the fluorescent lamp; if less than the threshold, regardless of whether the classroom someone, don't turn on the lights. (Figure 5)

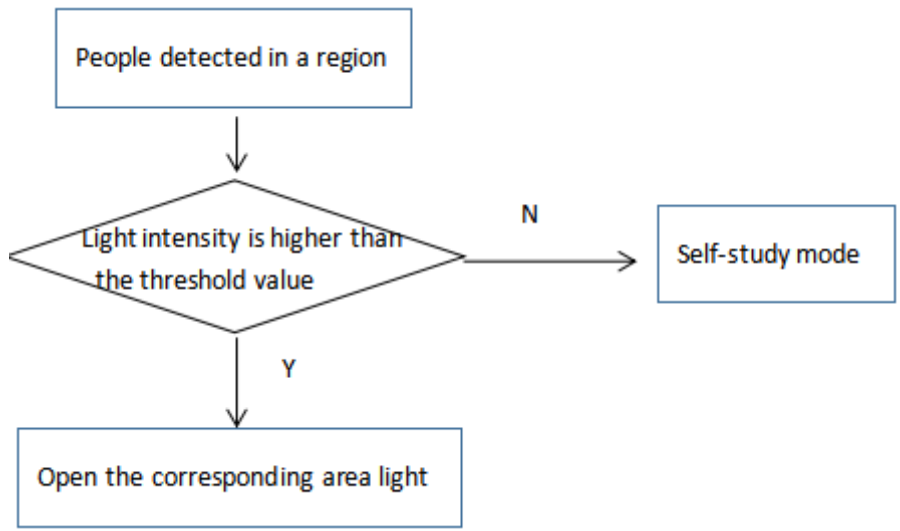

Fig.7 division diagram of the classroom

Because each classroom row of different courses, can be downloaded to the corresponding schedule different classrooms through the PC PC software, there will be no class and class were set to 1 and 0 , and stored in the computer. The fash machine is in regular working state, 5 minutes before the beginning of each class, teaching room the opportunity to make a judgment, if there is a corresponding class, open teaching mode, lights.

\section{Summary}

In order to realize the intelligent and electric energy utilization of classroom lighting control system, to achieve the goal of energy saving, The intelligent lighting system is designed.AT89C51 is the core of the control system. Classroom was divided into several regions. By scanning the signal of human body with infrared device periodically, the lights in the unmanned regions can be turned off automatically. And if students are too scattered, lights in some regions will also be turned off automatically to assemble the people. This system solved the problem of the much waste of university classroom lighting system and increased energy usage ratio significantly.

\section{Acknowledgments}

This work was financially supported by the Science and Technology Plan Projects of Qingdao Huanghai University.

\section{References}

[1] Lin Fangjian, Xu Bu-gong. Street light energy-saving control system based on zigBee network[J]. Control Engineering of China,2009,16 (3):324-328.

[2] Min Lijuan, Lu Hanhua, Wang Yashi.Design of host computer software in smart switch lighting control system[J].Computer Engineering,2011,37(22):290-294.

[3] Bi Xiaoguo,Liu Xudong, Niu Wei.Flame-fusion Growth of Rutile Single Crysta[J].Advanced Engineering Materials II,2012.5:535-537

[4] Gong Pan,Wang Jiamei, Sun Shantong. Design of intelligent lighting system based on ZigBee-DALI protocol[J].Modern Electronics Technique,2016,39 (4):63-66.

[5] Jiang Hongjiu, Gu Pengwei, Lu Yao,et al, The design of the intelligent illumination control system based on single chip microcontroller[J].Electronic Design Engineering,2015,23(23):107-109. 